\title{
Strike Out for Eight-Year Magnetic Monopole Search
}

\author{
No magnetic monopoles have been found in an analysis of data from the \\ IceCube Neutrino Observatory, but the analysis method could still help \\ advance the search for these elusive particles.
}

By Sarah Wells

ए xploring the push and pull of a bar magnet's polarity is among many children's first classroom exposure to physics. While dipole magnets-those with two poles-are ubiquitous on Earth and are used in everything from toys to particle colliders, other types of magnetic system are predicted to exist. For example, the Universe should also be abundantly filled with magnetic "monopoles," hypothetical particles with just one pole. These particles are predicted to have been produced during the big bang, but that prediction remains to be experimentally verified. Now, the collaboration behind the IceCube experiment has searched for these elusive particles in eight years of data collected by the IceCube Neutrino Observatory in Antarctica [1]. The collaboration came up empty handed, but its analysis could help in fine-tuning future magnetic monopole searches.

IceCube's 5000 sensors were designed to detect signs of neutrinos (see Viewpoint: Hunting the Sterile Neutrino), but

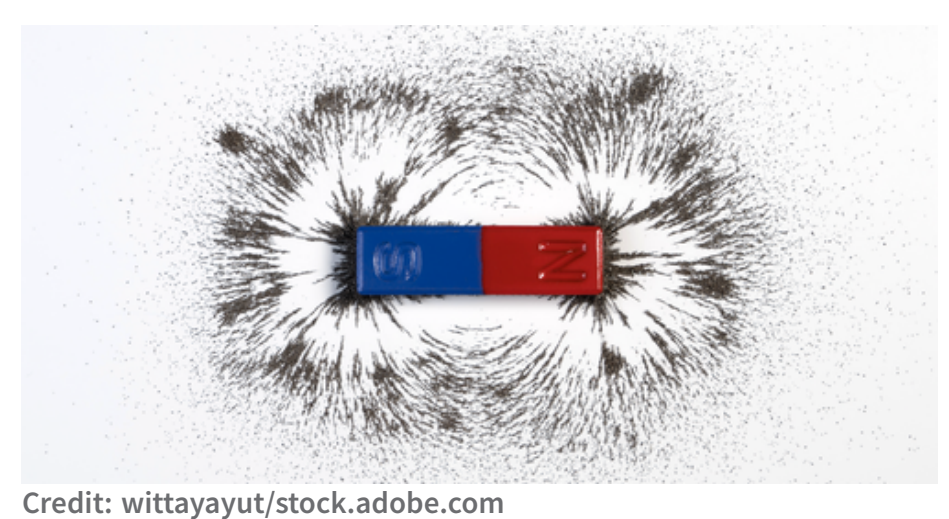

they should also be able to spot signals of magnetic monopoles. This ability arises because when monopoles pass through ice, they are expected to emit a form of radiation, known as Cherenkov radiation, that is also created when neutrinos interact with the same ice and which can be detected by IceCube's sensors.

To search for monopole signals, the team developed a machine-learning model to look for the very bright and very straight Cherenkov radiation emissions expected from monopoles traveling near the speed of light.

Ultimately, the team found no magnetic monopoles within the 2886 days of IceCube data that they evaluated. They did, however, place a new, stricter upper limit on the possible flux of magnetic monopoles.

Sarah Wells is a freelance science journalist based in Boston.

\section{REFERENCES}

1. R. Abbasi et al., "Search for relativistic magnetic monopoles with eight years of IceCube data," Phys. Rev. Lett. 128, 051101 (2022). 